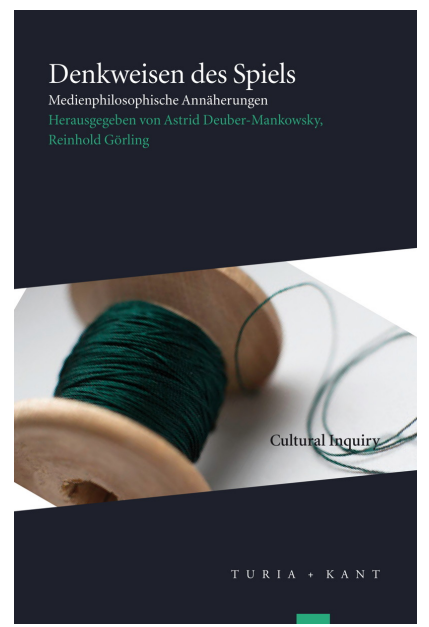

Denkweisen des Spiels: Medienphilosophische Annäherungen, hg. v. Astrid Deuber-Mankowsky und Reinhold Görling, Cultural Inquiry, 10 (Wien: Turia + Kant, 2017), S. 103-18

\section{JASMIN DEGELING \\ Über die Rhetorik des Spiels bei Michel Foucault}

\author{
ZITIERVORGABE:
}

Jasmin Degeling, »Über die Rhetorik des Spiels bei Michel Foucault «, in Denkweisen des Spiels: Medienphilosophische Annäherungen, hg. v. Astrid Deuber-Mankowsky und Reinhold Görling, Cultural Inquiry, 10 (Wien: Turia + Kant, 2017), S. 103-18 <https://doi.org/ 10.37050/ci-10_06>

ANGABE ZU DEN RECHTEN:

(C) by the author(s)

This version is licensed under a Creative Commons AttributionShareAlike 4.0 International License. 


\section{ÜBER DIE RHETORIK DES SPIELS BEI MICHEL FOUCAULT}

Jasmin Degeling

Es ist bemerkenswert, dass in so gut wie allen Texten und in unterschiedlichen begrifflichen Zusammenhängen in den Texten Michel Foucaults von "Spielen « die Rede ist: So ereignet sich der Diskurs der Diskursanalyse als Spiel, aber auch Machtbeziehungen produzieren sich in Wechselspielen und die Geschichte der Wahrheit lässt sich mit Foucault als Geschichte von »Spielen der Wahrheit « erzählen. So ergibt sich von der einfachen Beobachtung aus, dass in vielen Texten Foucaults eine prominente Metaphorik des Spiels auftaucht, die Notwendigkeit, sich die rhetorischen Funktionen und Verständnisweisen des Spiels (»jeu «) genauer anzuschauen, um damit der Frage Raum zu geben, ob es in den Denkweisen Foucaults eine spezifische Denkweise des Spiels gibt. Die Lektüren, die im Folgenden unternommen werden, weisen zunächst darauf hin, dass diese Frage kompliziert ist: Denn die Machtanalytik Foucaults leistet anstelle einer begrifflichen oder konzeptionellen Arbeit eher eine Metaphorik des Spiels und führt uns daher zu einer Diskussion der Rhetorizität Foucault'scher Denkweisen. Diese Spielmetaphern - und die Ausnahme hierzu bilden die "Spiele der Wahrheit", denn hier handelt es sich um einen Begriff -, scheinen eher marginal, und führen doch in die Zentren der Macht- und der Wahrheitsdiskurse.

Die vorsichtige Rekonstruktion der Spielbegriffe bei Foucault führt uns im Wesentlichen zu drei exemplarischen Schauplätzen einer Metapher des Spiels, die zunächst lokal untersucht werden: Foucaults Einführung in Kants Anthropologie (1961), Die Ordnung des Diskurses (1970) sowie Subjekt und Macht (1982). Als wichtigstes Instrument oder wichtigster Mitspieler in dieser Recherche erwies sich das Verhältnis von Übersetzungen zur Originalsprache, denn Übersetzungen leisten mitunter mittels der jeweiligen Übertragung des Spiels als rhetorischer Figur interessante Interpretationen und arbeiten somit ebenfalls an den latenten Denkweisen des Spiels mit, die die Texte und Konzepte Foucaults bearbeiten. ${ }^{1}$ Die folgenden Schauplätze sind in der chronologischen Rei-

1 Die Texte werden vornehmlich in der deutschen Übersetzung zitiert. Wenn es 
henfolge ihres Auftauchens in den Texten Foucaults geordnet - womit ich jedoch nicht unterstellen möchte, mit Blick auf die rhetorische Funktion des Spiels werde ein bislang unscheinbarer roter Faden sichtbar, der die ansonsten heterogenen Gegenstandsbereiche, deren Untersuchung sich Foucault bekanntlich methodisch je situativ gewidmet hat, endlich kohärent zu einem fertigen Werk vernähe. Vielmehr sind die folgenden Überlegungen das Ergebnis einer vorsichtigen Suche, die der Frage nachgeht, inwiefern Foucault'sche Konzepte anschlussfähig und produktiv sind für die Frage nach möglichen »Denkweisen des Spiels«.

\section{SPIELZEUG - EINFÜHRUNG IN KANTS ANTHROPOLOGIE (1961)}

Bereits in diesem frühen, 1968 geschriebenen, aber bis 2008 in Frankreich bzw. 2010 in Deutschland unveröffentlichten Text Foucaults, einer als »Einführung " gedachten Kommentierung von Kants zu jener Zeit noch wenig in der Forschung behandelter Anthropologie in pragmatischer Hinsicht, schenkt Foucault der Metapher des Spiels in Kants Text besondere Aufmerksamkeit:

Dieser Begriff des Spielens ${ }^{* 2}$ ist von besonderer Wichtigkeit: Der Mensch ist ein Spiel der Natur; aber dieses Spiel spielt er, und es spielt sich um ihn ab; und wenn ihm darin mitgespielt wird, wie in den Illusionen der Sinne, so hat er selbst gespielt, das Opfer dieses Spiels zu sein; während es ihm gebührt, Herr des Spiels zu sein und es im Kunstgriff einer Absicht auf seine Rechnung zu nehmen. ${ }^{3}$

sinnvoll ist, Differenzen zwischen verschiedenen Fassungen (bspw. Original und Übersetzung oder auch zwischen verschiedenen Übersetzungen) zur Kenntnis zu nehmen, werde ich diese ebenfalls angeben. Zu Rate gezogen wurden die französischen, deutschen und englischen gängigen Ausgaben. Ich verzichte aufgrund der gebotenen Kürze darauf, immer alle Fassungen in den verschiedenen Sprachen vollständig zu zitieren. Es zeigt sich hier übrigens ein hervorragendes Beobachtungsfeld für die Kontextabhängigkeit verschiedener Fassungen, die mitunter die Unterscheidung von Original und Übersetzung problematisieren.

2 Die Schreibweise entspricht dem Original. Foucault übernimmt Kants Spielbegriff und übersetzt ihn nicht ins Französische.

3 Michel Foucault, Einführung in Kants >Anthropologie (Frankfurt a.M.: Suhrkamp, 2010), S. 47. Vgl. auch Immanuel Kant, Anthropologie d'un point de vue pragmatique: précédé de Michel Foucault, introduction à l'anthropologie (Paris: Vrin, 2008), S. 33. 
Hier handelt es sich um ein opakes Spiel der Natur, das offenbar nicht auf Mitspieler_innen angewiesen ist und nach komplexen, unbekannten Regeln verfährt. Der Mensch dieser pragmatischen Anthropologie möge sich bemühen, dieses Spiel zu verstehen: Die Kant'sche Anthropologie fungiert als eine Art besonderer Ratgeber und unterrichtet eine Lehre vom »Können « und »Sollen «, die Aufschluss über die Möglichkeiten gibt, sich im Alltag als Mensch im Spiel der Natur zu üben.

[Die anthropologische Reflexion] wird den Sinn haben, den Menschen in dieses bildende Element zu versetzen. Sie wird also unauflösbar zugleich Analyse der Art sein, auf die der Mensch die Welt erwirbt (ihren Gebrauch, nicht ihre Kenntnis), das heißt, wie er sich in ihr installieren, ins Spiel eintreten und Mitspielen* kann, und Synthese der Vorschriften und Regeln, welche die Welt dem Menschen auferlegt, durch welche sie ihn bildet und in den Stand versetzt, das Spiel zu verstehen $* 4$

"Mitspielen « meint hier also den Versuch sich der Illusion des Mitspielen-Könnens (und Mitspielen-Sollens) zu bemächtigen und zwar in einem gegebenen Spiel, das mit keiner Spielerin zu rechnen scheint und dessen Regeln und Verfahrensweisen, Vollzüge und Dynamiken ein Rätsel sind, dessen Produkt man auch selbst zu sein scheint - sich hierin also wenigstens der Illusion des Mitspielen-Könnens (und MitspielenSollens) zu bemächtigen. Eine solche Lektüre der Kant'schen Anthropologie kann man sicher bestreiten: So hat zum Beispiel Astrid DeuberMankowsky darauf hingewiesen, dass die Vorstellung des Menschen als »Spielball« der Natur im Widerspruch zu Kants emphatischem Verständnis des Mitspielens als Möglichkeit zur Erziehung eines Weltbürgertums stehe. ${ }^{5}$ Bemerkenswert in Bezug auf Foucaults Lektüre des Spiels ist aber, dass selbst in dieser nietzscheanischen Version, in der die für Kant so wichtige Figur des Menschen nur noch als Spielzeug auftritt, Foucault diesem pragmatischen Denken viel abgewinnen kann. ${ }^{6}$ Denn die Pragmatik dieser Anthropologie fordert weniger die Erkenntnis der

4 Foucault, Einführung in Kants >Anthropologie<, S. 47-48. Das indirekte Zitat bezieht sich auf Immanuel Kant, Werkausgabe, hg. v. Wilhelm Weischedel (Frankfurt a.M.: Suhrkamp, 1977) XII: Schriften zur Anthropologie, Geschichtsphilosophie, Politik und Pädagogik 2, S. 400. Vgl. Kant, Anthropologie d'un point de vue pragmatique, S. 33.

5 Vgl. Astrid Deuber-Mankowsky, Praktiken der Illusion (Berlin: Vorwerk 8, 2007), S. 74-75.

6 Vgl. Foucault, Einführung in Kants >Anthropologie<, S. 63. 
Grenzen der Erkenntnis ein, wie es das kritische Projekt vorhat, sondern beschäftigt sich auf humorvolle und mitunter lakonische Weise mit dem »Gebrauch «: dem alltäglichen Handeln, den praktischen Spielräumen, die sich so für die Einzelnen ergeben könnten in Bezug auf das soziale Miteinander, und gibt sich somit weitgehend damit zufrieden, dass es sich hierbei um einen erkenntnistheoretisch nicht so abgesicherten Raum handelt. Tatsächlich gilt das Interesse, das diese Kant-Lektüre leitet und die wiederum aus meiner Sicht bereits früh in den Schriften Foucaults ein Denken des Spiel mitbefördert, gerade dieser Pragmatik des Übens, Einübens, Handelns und damit einer Dimension des Spiels, die sich in späteren Texten weiterverfolgen lässt und so etwas wie eine latente Denkweise des Spiels ausbildet.

\section{MITSPIELEN - DIE ORDNUNG DES DISKURSES (1970)}

Erstaunlicherweise diskutiert Die Ordnung des Diskurses verglichen mit Einführung in Kants Anthropologie besonders das Mitspielen: Der Diskursbegriff, dem diese Antrittsvorlesung gewidmet ist, stellt sich explizit - und besonders im französischen Original - über eine Metaphorik des Spiels und der Spielregel her, heißt es doch: »le discours n'est rien de plus qu'un jeu « ${ }^{7}$ bzw. »Der Diskurs ist immer nur ein Spiel. ${ }^{8}$ Ist die Diskursanalyse in der inzwischen sehr umfangreichen Rezeption stark verbunden mit der Frage, was vom Diskurs ausgeschlossen wird, zeigt die Spielmetaphorik noch einmal deutlich, dass dem Ausschluss das Thema des Eintretens in den Diskurs gleichwertig gegenübersteht. Versteht man als Spiel zunächst den Zusammenhang und die Vermittlung von Spielregeln, könnte man einen Diskurs ebenfalls vermittelt über eine Reihe von Regeln und ein intrinsisches Ordnungssystem, eine »innere Ökonomie «,

7 Michel Foucault, L'ordre du discours. leçon inangurale au Collège de France prononcée le 2 décembre 1970 (Paris: Gallimard, 1971), S. 52.

8 Michel Foucault, Die Ordnung des Diskurses, hg. Ralf Konersmann (Frankfurt a.M.: Fischer-Taschenbuch-Verlag, 1991), S. 32-33: »Ob es sich nun um eine Philosophie des begründenden Subjekts handelt oder um eine Philosophie der universellen Vermittlung - der Diskurs ist immer nur ein Spiel: ein Spiel des Schreibens im ersten Fall, des Lesens im zweiten oder des Tauschs im dritten. Und dieses Tauschen, dieses Lesen, dieses Schreiben spielen immer nur mit den Zeichen.«

$9 \quad$ Foucault, Die Ordnung des Diskurses, S. 44. 
bestimmen. Doch stellt sich dann erst recht die Frage, was unter einer Spielregel zu verstehen ist. Fragen wir daher umgekehrt: Welches Verständnis der (Spiel)Regel entwirft der Diskursbegriff? Gestalt gewinnt diese Frage gerade mit Blick auf den Beginn der Inauguralvorlesung und ihren theatralen Komponenten: Foucault als derjenige, der das Programm der Diskursanalyse zugleich herstellt und beschreibt, verhilft den Diskursen durch seine Analyse erst recht zu Recht und Realität. Foucault muss selbst mitspielen in diesem Spiel, das er zum Gegenstand der Analyse macht. Der Ort der Diskursanalyse gleicht daher Foucault zufolge einem Theater, ${ }^{10}$ das sich provisorisch einrichtet und in einem spielerischen Verhältnis zur Wirklichkeit steht. Wer allerdings auf der Bühne der Diskursanalyse einen Auftritt hat, kann nur noch schlecht behaupten, die Stimme, die er verlauten lässt, gehöre in Wahrheit nur dem Text an, den es dort aufzuführen gelte. So zeigt sich schon in dieser Szene des Auftritts vor dem Collège de France das Problem des Anfangens, das gerade sichtbar wird, indem es von der Institution feierlich und rituell begangen wird, wie Foucault lakonisch kommentiert. Die Inszenierung des Diskurses als Spiel, dessen spezifische Regeln und Bedingungen Gegenstand der Analyse sein sollen, setzt keine Vorstellung von Spielregeln im Sinne eines Regelkatalogs ein, der gleichzeitig wie eine Art Gebrauchsanweisung fungiert. Vielmehr zielt sie auf die Pointe, dass das Verständnis von Regeln (und damit sind an dieser Stelle Prozeduren, Strategien, Mechanismen mitgemeint) erst durch das Mitspielen produktiv wird. Wenn man an Spiele im engeren Sinn denken würde, hätten wir es hier wohl eher weniger mit Brett- oder Kartenspielen zu tun, sondern beispielsweise mit jenem Spiel um Blicken und Verstecken, in das (nicht nur) Kinder Passant_innen in Bussen oder Warteschlangen unversehens zu verwickeln suchen. In dem Moment, in dem man merkt, dass dem eigenen schweifenden Blick mit einem spielerischen Wegsehen oder Verstecken geantwortet wird, das Spiel um die Blicke also aufgenommen wurde, ist zunächst das eigene Wegsehen selbst Teil des Spiels. Um ein solches Spiel, dessen »Einsatz « oder »entrée « immer schon paradoxer Effekt des Mitspielens ist, scheint es mir hier zu gehen. Im Zentrum dieses Spiels steht nicht so sehr die Regel, verstanden als Satz, der einen bestimmten Inhalt aussagt, sondern die Regel im Sinne ihrer Wie-

10 Vgl. Foucault, Die Ordnung des Diskurses, S. 10; Foucault, L'ordre du discours, S. 10 . 
derholbarkeit, die immer die Form einer »Reaktualisierung « hat. ${ }^{11}$ Auf dieses Verständnis von Wiederholung verweist Foucault mit seiner Würdigung der literarischen Schreibweisen von Jorge Luis Borges, die aus Foucaults Perspektive mit den Regeln des Autor-Diskurses zu spielen beginnen: Borges Kurzgeschichte Pierre Menard, Autor des Don Quijote - eine als Literaturkritik formulierte Lobeshymne auf das Werk des fiktiven Autors Pierre Menard, dessen derartiges Bemühen um eine zeitgenössische Version des Don Quijote in der Kurzgeschichte als buchstäblich identische Wiederholung des Originaltextes von Cervantes gepriesen wird. »Spiel von der Art Borges als Kommentar, der nur wörtliche (aber feierliche und erwartete) Wiederholung dessen ist, was er kommentiert; oder Spiel einer Kritik, die endlos von einem Werk spricht, das gar nicht existiert. « ${ }^{12}$ Borges’ Technik wird hier verstanden als Mitspielen, das die Regel im Zuge ihrer Wiederholung variiert und parodiert. Dieses Verständnis eines Spiels, das durch die Wiederholbarkeit seiner Regeln und Handlungen "eingesetzt " wird, dient Foucault zufolge dem Ausschalten des Zufalls, dem Bändigen des Chaos und der Kräfte des Außen. ${ }^{13}$ Die Metapher des Spiels ermöglicht hier also den Diskurs nicht einfach als gegebenen Regelkatalog und Regelungszusammenhang zu verstehen, sondern dieses Verständnis zurückzuführen auf ein Denken des Zufalls und des Ereignisses, des "Aleatorischen ", wie es im Französischen heißt und worin sich etwas genuin Spielerisches zeigt. ${ }^{14}$ In zweiter Instanz begreift die Metapher des Spiels das Mitspielen-Können und MitspielenSollen als diskursive Möglichkeitsbedingung überhaupt: Spiel steht also auch in Zusammenhang mit dem, was Foucault unter Wahrheit versteht.

Das Wort 'Spiek kann Sie zu einem Irrtum führen. Wenn ich von `Spiek spreche, dann spreche ich von einer Gesamtheit von Regeln zur Herstellung der Wahrheit. Dies bedeutet nicht Spiel im Sinne von Nachahmung

11 Vgl. Foucault, Die Ordnung des Diskurses, S. 25; Foucault, L'ordre du discours, S. 38 .

12 Foucault, Die Ordnung des Diskurses, S. 18; Foucault, L'ordre du discours, S. 25.

13 Vgl. bspw. Foucault, Die Ordnung des Diskurses, S. 25; Foucault, L'ordre du discours, S. 38. Man könnte hier auch an den Begriffs des Ritornells denken, den Deleuze und Guattari entwickelt haben in Bezug auf die beiden Aspekte des Rhythmus und der Bändigung des Außen, vgl. Gilles Deleuze u. Félix Guattari, Tausend Plateaus. Kapitalismus und Schizophrenie (Berlin: Merve, 2010), S. 474.

14 Vgl. Foucault, L'ordre du discours, S. 11 und 58. 
oder Schauspiel; es besteht in einer Gesamtheit von Verfahren, die zu einem bestimmten Resultat führen, das nach Maßgabe seiner Prinzipien und Verfahrensregeln als gültig oder ungültig, als erfolgreich oder als erfolglos betrachtet werden kann. ${ }^{15}$

Mitspielen heißt hier »im Wahren zu sein $",{ }^{16}$ die diskursiven Möglichkeitsbedingungen zu erfüllen, zu sagen, was gesagt werden kann - und nur, indem mitgespielt wird und mit den Regeln gespielt wird, rührt man an die Möglichkeitsbedingungen des Diskurses. Dieser Gedanke findet sich beim späten Begriff des Wahrheitsspiels/»jeux de la vérité « noch:

Der Herrschaft einer Wahrheit entkommt man also nicht, indem man ein Spiel spielt, das dem Spiel der Wahrheit vollständig fremd ist, sondern indem man das Wahrheitsspiel anders spielt, indem man ein anderes Spiel, eine andere Partie oder mit anderen Trümpfen spielt. ${ }^{17}$

Diese Rede vom Spiel bildet einen Resonanzraum der Sprachspiele, wie sie Ludwig Wittgenstein beschrieben hat; ${ }^{18}$ und sicher kann man auch die Rede vom Einbruch des Diskurses in die "jeux de la pensée et de la langue «, von welchen die deutsche Übersetzung nur den »Einbruch [des Diskurses] in Sprache und Denken« übrig lässt, vor dem Hintergrund

15 Michel Foucault, "Die Ethik der Sorge um sich als Praxis der Freiheit ", in Ästhetik der Existenz. Schriften zur Lebenskunst (Frankfurt a.M.: Suhrkamp, 2007), S. 274. Vgl. Michel Foucault, Dits et écrits, tome 4 (Gallimard: Paris, 1994), S. 725.

16 Foucault, Die Ordnung des Diskurses, S. 24 und 36.

17 Foucault, "Die Ethik der Sorge um sich als Praxis der Freiheit«, S. 253-80 (S. 272). In der Originalsprache heißt es: »On échappe donc à une domination de vérité, non pas en jouant un jeu totalement étranger au jeu de la vérité, mais en le jouant autrement ou en jouant un autre jeu, une autre partie, d'autre atouts dans le jeu de vérité." (Foucault, Dits et écrits, tome 4, S. 724).

18 Das Verhältnis von Foucault'schen Wahrheits- und Wittgenstein'schen Sprachspielen ist bereits häufig Gegenstand der Forschung gewesen. Hier sei exemplarisch verwiesen auf: Foucault, Wittgenstein: de possibles rencontres, hg. v. Frédéric Gros u. Arnold I. Davidson (Paris: Kimé, 2011); Jörg Volbers, Selbsterkenntnis und Lebensform: Kritische Subjektivität nach Wittgenstein und Foucault (Bielefeld: transcript, 2008); Axel Honneth, »Foucault und die Humanwissenschaften. Zwischenbilanz einer Rezeption ", in Michel Foucault: Zwischenbilanz einer Rezeption. Frankfurter Foucault-Konferenz 2001, hg. v. Axel Honneth; Martin Saar (Frankfurt a.M.: Suhrkamp, 2001), S. 15-26. 
des Begriffs der »Sprachspiele « verstehen. ${ }^{19}$ Was ist ein Sprachspiel? Wittgensteins Liste von Beispielen für solche könnte auch im Zusammenhang jener mutmaßlichen chinesischen Enzyklopädie auftauchen, die Borges in Die analytische Sprache von John Wilkins vermeintlich zitiert, und die bei Foucault im Vorwort zu Die Ordnung der Dinge auftaucht, die Leistung würdigend, das Monströse jeder Taxonomie zur Darstellung gebracht zu haben:

23. Es gibt unzählige solcher Arten [von Sätzen, d. Verf.]: unzählige verschiedene Arten der Verwendung alles dessen, was wir 'Zeichen', ,Worte‘, Sätze`, nennen. Und diese Mannigfaltigkeit ist nichts Festes, ein für allemal Gegebenes; sondern neue Typen der Sprache, neue Sprachspiele, wie wir sagen können, entstehen und andere veralten und werden vergessen. [...] Das Wort Sprachspiek soll hier hervorheben, daß das Sprechen der Sprache ein Teil ist einer Tätigkeit, oder einer Lebensform. Führe dir die Mannigfaltigkeit der Sprachspiele an diesen Beispielen, und anderen, vor Augen:

Befehlen, und nach Befehlen handeln -

Beschreiben eines Gegenstands nach dem Ansehen, oder nach Messungen -

Herstellen eines Gegenstands nach einer Beschreibung (Zeichnung) -

Berichten eines Hergangs -

Über den Hergang Vermutungen anstellen -

Eine Hypothese aufstellen und prüfen -

Darstellen der Ergebnisse eines Experiments durch Tabellen und Diagramme -

Eine Geschichte erfinden, und lesen -

Theater spielen -

Reigen singen -

Rätsel raten -

Einen Witz machen; erzählen -

Ein angewandtes Rechenexempel lösen -

Aus einer Sprache in eine andere übersetzen -

Bitten, Danken, Fluchen, Grüßen, Beten. ${ }^{20}$

Wittgenstein versteht Sprache radikal vom Sprechen her, von ihrem Gebrauch - einem der wichtigsten Begriffe, der hier mitarbeitet und der

19 Foucault, Die Ordnung des Diskurses, S. 16; Foucault, L'ordre du discours, S. 33.

20 Ludwig Wittgenstein, Werkausgabe in acht Bänden, hg. v. Joachim Schulte (Frankfurt a.M.: Suhrkamp, 2001), I: Kritisch-genetische Edition, S. 758-59. 
bereits, wie ich zu zeigen versuche, in Zusammenhang mit Kants Anthropologie einflussreich ist. Wittgenstein sucht hier jede abstrakte, logische oder klassisch strukturalistische Klassifikation zurückzuweisen und stellt die Sprachspiele der Vorstellung entgegen, Sprache sei erfasst durch das Verhältnis von Wort und Bezeichnung. Spiele werden verstanden als Ähnlichkeitsbeziehungen, deren Charakteristiken mitnichten vermittels der Regelsysteme erfasst werden können. ${ }^{21}$ Das Sprachspiel ist definiert als Sprache, verwoben mit ihren Tätigkeiten: ${ }^{22}$ Diese Pragmatik weist durchaus eine Ähnlichkeit auf zur Vorstellung, dass das Verhältnis von Dingen zu Ordnungssystemen eine Konsistenzebene bildet, die ihre eigenen Geschichten und Episteme ausbildet. Diese Verwobenheit von Sprachspielen bzw. Gebrauchsweisen und Dingen ist bei Wittgenstein verbunden mit der Ausbildung von »Lebensformen «. So könnte man vor diesem Hintergrund das für Foucault gerade in den späten Schriften so wichtige Verhältnis von Diskursen, Wahrheitsspielen und Lebensformen » in pragmatischer Hinsicht « - augenzwinkernd formuliert - über das Erbe einer Rhetorik des Spiels über Wittgenstein skizzieren.

STRATEGIESPIELE - SUBJEKT UND MACHT (1982)

Scheint sich bis hierhin eine Linie anzudeuten, die den Spielbegriff als »Kategorie der Handlung « durch Foucault hindurch führt und sogar philosophische Abstammungslinien im »pragmatischen « Denken behauptet - ergibt sich in Hinblick auf das Verhältnis von Spiel und Macht eine feine Differenz, die die systematische Gleichsetzung von »Spielen der Macht « mit »Spielen der Wahrheit « unterläuft: Indem Foucault Machtbeziehungen im Verhältnis zu »Strategien « (Spielstrategien wie Kriegsstrategien) diskutiert, ist damit nicht allein die »Gesamtheit von Verfahren, Prozeduren, Regeln « gemeint, vielmehr sind Machtbeziehungen eine besondere »Form handelnder Einwirkung auf andere " (Frz.: »c'est un mode d'action de certains sur certains autres «). ${ }^{23}$ Der Begriff der Handlung gewinnt hier schärfere Kontur über sein Spannungsverhältnis zur Strategie. Der 1982 zuerst in englischer Sprache durch Paul Rabinow und Hubert Dreyfus als Nachwort zu deren Buch

\footnotetext{
21 Vgl. Wittgenstein, Philosophische Untersuchungen, S. 787.

22 Vgl. ebd., S. 749.

23 Michel Foucault, »The Subject and Power «, Critical Inquiry, 8.4 (1982), S. 77795 (S. 795); Foucault, Dits et écrits, tome 4, S. 235-36.
} 
Michel Foucault - Beyond Structuralism and Hermeneutics veröffentlichte Aufsatz The Subject and Power diskutiert den späten Machtbegriff Foucaults in konzentrierter Form: Entscheidend für das, was mit den Machtbeziehungen rauf dem Spiel steht‘, scheint eine Problematisierung der diesen inhärenten Zwecke zu sein, das heißt der Finalitäten, deren Schauplatz der Aushandlung Machtgefüge bilden. Foucault spricht tatsächlich von einem »Handlungsfeld «, das die Machtbeziehungen bilden, das immer auch ein "open field of possibilities « sei. ${ }^{24} \mathrm{Im}$ Fluchtpunkt der Differenz von Machtbeziehungen und Machtstrategien steht eine kritische Diskussion der Zweck-Mittel-Beziehungen, die Machtbeziehungen einrichten. ${ }^{25}$ Die folgende Definition, die im Text unter der Frage, »Wie wird Macht ausgeübt «, auftaucht, liefert eine Bestimmung der Strategie im Verhältnis zum Spiel. Allerdings setzt die deutsche Übersetzung von Michael Bischoff an die Stelle des Spiels ("game«) die Spieltheorie - und liefert mit dieser Interpretation einen deutlichen Hinweis auf die wissensgeschichtlichen Zusammenhänge, die die Problematisierung von Machtbeziehungen hier bedingt:

Der Ausdruck 'Strategie` wird gewöhnlich in drei Bedeutungen gebraucht. Erstens bezeichnet er die Wahl der für ein Ziel eingesetzten Mittel. In dieser Bedeutung verweist der Ausdruck auf Zweckrationalität. Zweitens bezeichnet man damit in der Spieltheorie ein Verhalten, bei dem die Partner ihr Verhalten auf das erwartete Verhalten der anderen und auf die eigenen Erwartungen hinsichtlich der Erwartungen der anderen abstellt. Hier geht es also letztlich um den Versuch, Einfluss auf andere zu nehmen. Und drittens bezeichnet der Ausdruck ein Verfahren, die in einer Auseinandersetzung eingesetzt werden, um den anderen seiner Kampfmittel zu berauben und ihn zur Aufgabe des Kampfes zu zwingen. Hier geht es also um die Mittel, die dazu dienen, den Sieg zu erringen. Diese drei Bedeutungen vereinigen sich in der Kampfsituation Krieg oder Spiel (frz. sguerre ou jeus) - , in denen es darum geht, auf einen Gegner so einzuwirken, dass der Kampf für ihn unmöglich wird. ${ }^{26}$ [Herv. d. Verf.]

24 Foucault, »The Subject and Power«, S. 789.

25 Vgl. hierfür auch das Interview von 1977 mit dem Titel: »Pouvoirs et stratégies «, in welchem Foucault in analoger Weise Machtbeziehungen und Strategien differenziert, um deren inhärenter »Spielräume« (frz. »marges «) Rechnung zu tragen. Vgl. Michel Foucault, Dispositive der Macht. Über Sexualität, Wissen und Wahrheit (Berlin: Merve, 1978), S. 210-12.

26 Michel Foucault, "Subjekt und Macht", in Ästhetik der Existenz. Schriften zur 
Unter Strategie kann man also eine im alltäglichen Sinn zweckbestimmte Handlung verstehen, sowie eine solche, wie sie in der Spieltheorie zum Schlüsselbegriff wird, ${ }^{27}$ nämlich als Handlung, die Effekt einer Antizipation der Zwecke ihrer Einwirkung ist; und schließlich kann Strategie eine kriegerische Bedeutung haben, wobei der Text hier darüber Aufschluss gibt, dass diese Bedeutung gleichermaßen im Spiel wie im Krieg zum Tragen kommt. Bemerkenswert ist, und das dürfte diese deutsche Übersetzung motiviert haben, dass im Englischen sogar von "game " die Rede ist anstelle der viel häufigeren Verwendung von "play « bei Foucault. Im selben Text grenzt Foucault das Spiel (»play«) in der allgemeineren Verwendung eines »Ensembles « sich wechselseitig bedingender Handlungen ab vom Spiel als "game«. Bemerkenswerterweise wird die Konnotation des Spiels als Nullsummenspiel (»zero-sum-game«) ausdrücklich zurückgewiesen ${ }^{28}$ - eine Beiläufigkeit, die die deutsche Übersetzung wiederum vermissen lässt. Gemeint ist hier das Nullsummenspiel als erstes Wissensobjekt der Spieltheorie. Dessen Erfinder John von Neumann gelang damit 1928 die erste mathematische Formalisierung eines spieltheoretischen Problems: Angenommen, die `Summe`, der der Einsatz gilt, ist konstant, wie etwa im Schach (das lediglich einen Sieg

Lebenskunst (Frankfurt a.M.: Suhrkamp, 2007), S. 81-104 (S. 102). »The word sstrategy is currently employed in three ways. First, to designate the means employed to attain a certain end; it is a question of rationality functioning to arrive at an objective. Second, to designate the manner in which a partner in a certain game acts with regard to what he thinks should be the action of the others and what he considers the others think to be his own; it is the way in which one seeks to have the advantage over others. Third, to designate the procedures used in a situation of confrontation to deprive the opponent of his means of combat and to reduce him to giving up the struggle; it is a question, therefore, of the means destined to obtain victory." [Herv. d. Verf.] (Foucault, »The Subject and Power «, S. 793). Im französischen Original: »[...]Pour désigner la manière dont un partenaire, dans un jeu donné, agit en fonction de ce qu'il estime que les autres penseront être la sienne; en somme, la manière dont on essaie d'avoir prise sur l'autre.« [Herv. d. Verf.] (Foucault, Dits et écrits, tome 4, S. 240).

27 Vgl. Claus Pias, "Entscheide dich!: Was uns entscheidet: Krieg und Spiele«, in Neue Rundschau, Band 113/2 (Frankfurt a.M.: S. Fischer, 2002), S. 28-43 <http://fox.leuphana.de/portal/files/530182/rundschau.pdf> [Zugriff: 17.08.2015].

28 Vgl. Foucault, »Subjekt und Macht«, S. 92-93; Foucault, »The Subject and Power $«$ S. 784. 
kennt, dem die Niederlage des zweiten Spielers gegenübersteht, wohingegen im Lotto die Summe jedes Mal von Anzahl und Einsatz der Mitspieler_innen abhängt), werden Berechnungen darüber angestellt, welche Gewinnverteilungen möglich sind. Ich denke, dass Foucault hier die Analogie zum Nullsummenspiel zurückweist, die sich aufdrängt, wenn Machtbeziehungen als strategische Beziehungen zwischen Partnern bestimmt werden, um der Vorstellung Einhalt zu gebieten, Macht sei eine konstante Größe, deren Verteilung berechnet werden könne. Dann käme die Analyse von Machtbeziehungen einem Nullsummenspiel gleich. Hier besteht ein Unterschied zur Spieltheorie, die Verhalten formalisiert, indem sie sich auf die Annahme stützt, dass Entscheidungen situativ autonom und mit dem Zweck des größten anzunehmenden Nutzen getroffen würden. Im Sinne der Spieltheorie wird also im Zweifelsfall der spielerische Aspekt kassiert, weil die Zweckrationalität einer Handlung das Element ist, das die Berechenbarkeit gewährleistet. Dass zwar plötzlich explizit die Spieltheorie in der gängigen deutschen Übersetzung genannt wird, aber das Argument, Macht sei nicht mit einem Nullsummenspiel gleichzusetzen, seltsamerweise getilgt ist, mag als Symptom dafür gelten, dass innerhalb der deutschen Rezeption bisher wenig Sensibilisierung für das spielerische Element der Denkweisen Foucaults stattgefunden hat. Foucault unterscheidet noch einmal zwischen dem strategischen Aspekt einer Handlung, also ihrem Zweck, und der Relationalität der Handlungen zueinander, die überhaupt erst Macht als Beziehung herstellen, und innerhalb derer die Strategien miteinander »ins Spiel « treten. Der spielerische Aspekt der Machtbeziehungen wird sogar als »free play « bezeichnet. "Statt die Macht im Blick auf innere Rationalität zu analysieren, möchte ich die Machtbeziehungen über das Wechselspiel gegensätzlicher Strategien untersuchen. ${ }^{29}$

"Innere Rationalität " wird nicht zum Standpunkt der Analyse von Machtbeziehungen gemacht, denn das würde implizieren, die ZweckMittel-Beziehungen zum entscheidenden Kriterium zu machen. Stattdessen sollen antagonistische Verhältnisse, Wechselspiele, Relationen Ausgangspunkt der Analyse von Macht sein, und deswegen taucht der Begriff »Macht « kaum noch auf, sondern nur » Machtbeziehungen«.

29 Foucault, »Subjekt und Macht«, S. 84. »Rather than analyzing power from the point of view of its internal rationality, it consists of analyzing power relations through the antagonism of strategies« (Foucault, »The Subject and Power«, S. 780). 
Zwar führt der Begriff der Machtbeziehungen die Rhetorik des Spiels mit, wie sie etwa in Die Ordnung der Dinge auftaucht, erweitert diese aber um das Spiel/»game « der Spieltheorie und konkretisiert damit den Handlungsbegriff. Machtbeziehungen sind ausdrücklich nur über ihren Handlungsaspekt beschreibbar: Das semantische Feld der Handlung als Übung und Ausübung (exercise bzw. exercice) arbeitet daran mit. Die Pointe, die diese Charakterisierung des Spiels für den Machtbegriff bedeutet, ist, dass Freiheit (»freedom «) als Bedingung der Möglichkeit der Machtbeziehungen als Spiel fungiert. ${ }^{30}$ Foucault sagt ganz einfach: Wenn Macht (strategisch) handelndes Ausüben auf andere Handlungen ist, dann ermöglicht dieses Verhältnis der Handlungen zueinander ein irreduzibles Moment der Freiheit. Dieser Handlungsbegriff heißt dann $»$ Regierung «/»government $"$.

The exercise of power is not simply a relationship between partners, individual or collective; it is a way in which certain actions modify others. [...]

When one defines the exercise of power as a mode of action upon the actions of others, when one characterizes these actions by the government of men by other men - in the broadest sense of the term - one includes an important element: freedom. Power is exercised only over free subjects, and only insofar as they are free. By this we mean individual or collective subjects who are faced with a field of possibilities in which several ways of behaving, several reactions and diverse comportments, may be realized. ${ }^{31}$

Über diese Semantik des Übens und Ausübens wird jedoch gerade problematisch, wie Foucault das Subjekt bzw. die Position dessen, was als Subjekt auftaucht, adressiert, verbindet doch Foucault diesen Machtbegriff der Regierung direkt mit dem »freien Subjekt « und geht es doch gerade in diesem späten Aufsatz nicht nur um eine Klärung der Machtanalytik, sondern um die Beziehung von Subjekt und Macht. Daher expliziert der Text, dass die Richtung der Fragen Foucaults nie durch das Problem der Macht bestimmt gewesen sei, ${ }^{32}$ vielmehr führe ja gerade

Vgl. Foucault, »The Subject and Power «, S. 790.

31 Ebd., S. 788-90.

32 Vgl. Foucault, "Subjekt und Macht«, S. 81. »Thus, it is not power but the subject which is the general theme of my research.« (Foucault, »The Subject and Power«, S. 778). 
die Problematisierung der Objektivierungstechniken des Subjekts - etwa zum Irren, zum Kranken, zum Delinquenten - zur Analytik der Machtbeziehungen. In der späten Fassung sind diese Machtbeziehungen spezifisches Element der Wahrheitsspiele, ein weiteres, das mit diesen in spielerischer Wechselwirkung steht, sind die Selbsttechniken, bekanntlich als Instrumente der Selbsttransformation des Subjekts verstanden..$^{33}$ Somit steht hier die Politisierung des Problems der Handlungsmacht auf dem Spiel. Wie aber wird das Verhältnis von Handlungsmacht und Subjekt bestimmt und inwiefern spielt der Begriff der Selbsttechniken dabei eine Rolle?

Foucault situiert Machtbeziehungen als Spiel der Kräfteverhältnisse, um Praktiken des Widerstands ein Handlungsfeld zu geben. Damit ist ein weiteres wichtiges Argument dafür, die Zweckrationalität von Handlungen nicht als alleiniges Element von strategischen Machtspielen zu verstehen, eingeführt: Widerstand ist immer zweckgerichtet, weil er sich immer gegen ein spezifisches Verhältnis richtet. Das »freie Spiel « der Machtbeziehungen im Zeitalter der Regierung ist für Foucault immer auch eines »transversaler Kämpfe « ${ }^{34}-$ und hier klingt noch einmal eine Rhetorik nach Guattari/Deleuze an. ${ }^{35}$

Hierfür lohnt es sich, Christoph Menkes einflussreiche Lektüre des Subjektmodells Foucaults zur Kenntnis zu nehmen, um die Frage nach dem Verhältnis von Subjekt und Handlungsmacht noch einmal zu schärfen. Menkes Schlüsselbegriff ist die »Übung « - womit das virulente, nämlich pragmatische Element des Machtbegriffs fokussiert wird, nämlich Handeln/Ausüben/Üben/Einüben bzw. exercise/exercice, das die Semantik des Spiels durch die Texte hindurch mitführt - aber erweitert um die Bedeutung des »Sich-Führens «:

Subjektivität ist das praktische Selbstverhältnis des Sich-Führens, das seinen Ort im Etwas-Ausführen hat. [...] Subjektsein heißt Machthaben im genannten doppelten Sinn: das Gut einer Tätigkeit oder Praxis erwir-

Vgl. Michel Foucault, »Technologien des Selbst«, in Schriften in vier Bänden. Dits et Ecrits, (Frankfurt a.M.: Suhrkamp, 2005) IV: 1980-1988, S. 966-98.

34 Foucault, »Subjekt und Macht«, S. 85; Foucault, »The Subject and Power«, S. 780 .

35 M. E. arbeitet diese ebenfalls an der Rede vom »individuellen wie kollektiven Subjekt« mit. Vgl. Gilles Deleuze, »Vorwort. Drei Gruppenprobleme«, in Die einsame Insel. Texte und Gespräche 1953-1974 (Frankfurt a.M.: Suhrkamp, 2003), S. 282-97. 
ken zu können, weil man seine eigenen Bewegungen entsprechend zu führen vermag. ${ }^{36}$

Aus dieser Perspektive rekurriert die Foucault'sche Übung auf ein Subjektmodell, das mindestens ab Überwachen und Strafen ontologisch dasselbe sei. ${ }^{37}$ Subjektivierung wird im » Medium der Übung « verortet, und damit fällt der Begriff der Übung hier dem Bereich der Selbsttechnik zu. Entscheidend ist in dieser Lektüre die Frage, wann Übungen Medien der Disziplinierung, wann Medien der Entunterwerfung seien, die bei Foucault unter dem Begriff Ästhetik der Existenz fungiere - beide Subjektivierungsweisen verhalten sich als Kippfigur zueinander, ${ }^{38}$ beide sind dennoch auch bei Menke nicht durch ihre Finalitäten bestimmbar. Jedoch privilegiert Menke die Handlungsmacht vor der Freiheit; ${ }^{39}$ praktisches Handeln-Können sei gebunden an das Subjekt. Der Unterschied zur Foucault'schen Version des Machtbegriffs, die aus meiner Sicht die Bestimmung des freien Subjekts als Effekt von »Spielen der Macht « im skizzierten Sinn leistet, meint Menke mit Handlungsmacht die Macht des Subjekts, handeln zu können (selbst wenn das Handeln der Unterwerfung und Disziplinierung dient). An die Stelle einer Autonomie der Handlung tritt die Autonomie des Subjekts und kassiert damit das Spielerische, das, wie ich es hier zu zeigen versucht habe, mit einer Vorstellung freier Handlung einhergeht.

Ob Handlung im Sinne der vorgestellten Lektüre als Medium der Regierung bestimmt wird, sodass Ausüben, Üben, Einüben Wirkungszusammenhänge zwischen Handlungen bestimmen und Handlungsmacht die Macht der Handlung meint, oder, der Lektüre von Christoph Menke folgend, die Handlungsmacht ontologisch an das Subjekt zurück gebunden wird und damit die Macht zu handeln bezeichnet - diese oder jene systematische Bestimmung realisiert vermutlich unterschiedliche Spielweisen und Strategien im Spiel der Wahrheit.

36 Christoph Menke, »Zweierlei Übung. Zum Verhältnis von sozialer Disziplinierung und ästhetischer Existenz«, in Michel Foucault: Zwischenbilanz einer Rezeption. Frankfurter Foucault-Konferenz 2001, hg. v. Axel Honneth (Frankfurt a.M.: Suhrkamp, 2003), S. 283-99 (S. 286). Der Begriff der Übung wird Textstellen entnommen, die im Französischen »exercice « und »apprentissage « lauten.

37 Vgl. ebd., S. 285.

38 Vgl. ebd.

39 Vgl. ebd., S. 288. 
Vom Auftauchen des Spielzeugs* in den frühen Schriften Foucaults, über die Metapher der Spielregel des Diskurses bis hin zur späten Fassung des Macht- sowie Wahrheitsbegriffs bildet das, was Foucault (rhetorisch) miteinander ins Spiel bringt, eine erstaunliche Konsistenz aus. Übersetzungen sind hierfür mitunter nicht so sensibilisiert - jedenfalls übertragen gerade die deutschen Übersetzungen tendenziell diese latente Denkweise des Spiels nicht, die mir gerade in ihrer Rhetorizität interessant zu sein scheint. Es ist ein Element sprachlicher Konsistenz in den Schriften, das konzeptionell an den Begriffen mitarbeitet. Womit umso weniger ausgeschlossen ist, dass es sich um eine Konsistenz handelt, die eine Geschichte hat. Monique David-Ménard hat während unseres Workshops die Frage aufgeworfen, ob die merkwürdige Insistenz des Spiels im Denken Foucaults womöglich die letzte, latente Brücke zum Denken des philosophischen Idealismus aufrecht erhält - wenn man es sonst gerade als Anstrengung, dem Denken die idealistische Tendenzen auszutreiben, beschreiben kann. Diese Frage bleibt an dieser Stelle diskussionswürdig. 
|Jasmin Degeling, »Über die Rhetorik des Spiels bei Michel Foucault «, in Denkweisen des Spiels: Medienphilosophische Annäherungen, hg. v. Astrid Deuber-Mankowsky und Reinhold Görling, Cultural Inquiry, 10 (Wien: Turia + Kant, 2017), S. 103-18 <https: //doi.org/10.37050/ci-10_06>

\section{QUELLENANGABEN}

Deleuze, Gilles, »Vorwort. Drei Gruppenprobleme «, in Die einsame Insel. Texte und Gespräche 1953-1974 (Frankfurt a.M.: Suhrkamp, 2003), S. 282-97

Deleuze, Gilles und Félix Guattari, Tausend Plateaus. Kapitalismus und Schizophrenie (Berlin: Merve, 2010)

Deuber-Mankowsky, Astrid, Praktiken der Illusion (Berlin: Vorwerk 8, 2007)

Foucault, Michel, Dispositive der Macht. Über Sexualität, Wissen und Wahrheit (Berlin: Merve, 1978)

- Dits et écrits, tome 4 (Gallimard: Paris, 1994)

_ Einführung in Kants > Anthropologie< (Frankfurt a.M.: Suhrkamp, 2010)

L'ordre du discours. leçon inaugurale au Collège de France prononcée le 2 décembre 1970 (Paris: Gallimard, 1971)

—Die Ordnung des Diskurses, hg. Ralf Konersmann (Frankfurt a.M.: Fischer-TaschenbuchVerlag, 1991)

$\longrightarrow \gg$ Die Ethik der Sorge um sich als Praxis der Freiheit «, in Ästhetik der Existenz. Schriften zur Lebenskunst (Frankfurt a.M.: Suhrkamp, 2007), S. 274

— 448181>

— $\gg$ Subjekt und Macht «, in Ästhetik der Existenz. Schriften zur Lebenskunst (Frankfurt a.M.: Suhrkamp, 2007), S. 81-104

— $\gg$ Technologien des Selbst «, in Schriften in vier Bänden. Dits et Ecrits, (Frankfurt a.M.: Suhrkamp, 2005) IV: 1980-1988, S. 966-98

Gros, Frédéric und Arnold I. Davidson (Hg.), Foucault, Wittgenstein: de possibles rencontres (Paris: Kimé, 2011)

Honneth, Axel, »Foucault und die Humanwissenschaften. Zwischenbilanz einer Rezeption «, in Michel Foucault: Zwischenbilanz einer Rezeption. Frankfurter Foucault-Konferenz 2001, hg. v. Axel Honneth; Martin Saar (Frankfurt a.M.: Suhrkamp, 2001), S. 15-26

Honneth, Axel (Hg.), Michel Foucault: Zwischenbilanz einer Rezeption. Frankfurter FoucaultKonferenz 2001 (Frankfurt a.M.: Suhrkamp, 2003)

Kant, Immanuel, Anthropologie d'un point de vue pragmatique: précédé de Michel Foucault, introduction à l'anthropologie (Paris: Vrin, 2008)

Werkausgabe, hg. v. Wilhelm Weischedel (Frankfurt a.M.: Suhrkamp, 1977) XII: Schriften zur Anthropologie, Geschichtsphilosophie, Politik und Pädagogik 2

Pias, Claus, »Entscheide dich!: Was uns entscheidet: Krieg und Spiele «, in Neue Rundschau, Band 113/2 (Frankfurt a.M.: S. Fischer, 2002), S. 28-43 <http://fox.leuphana.de/portal/ files/530182/rundschau.pdf > [Zugriff: 17.08.2015]

Volbers, Jörg, Selbsterkenntnis und Lebensform: Kritische Subjektivität nach Wittgenstein und Foucault (Bielefeld: transcript, 2008) <https://doi.org/10.14361/9783839409251>

Wittgenstein, Ludwig, Werkausgabe in acht Bänden, hg. v. Joachim Schulte (Frankfurt a.M.: Suhrkamp, 2001), I: Kritisch-genetische Edition 